\title{
Preparation of Microstructured Surface of Pyrolytic Carbon by Laser and Its Blood Compatibility Application
}

\author{
Huan Feng ${ }^{1}$, Xia Ye ${ }^{* 1}$, Lei Lu ${ }^{1}$, Sheng $\mathrm{Xu}^{1}$, Xiaohong Yang ${ }^{2}$, Zhenmin Fan ${ }^{1}$, Che Yang ${ }^{1}$ and Jiabai Zhu ${ }^{1}$ \\ ${ }^{1}$ Department of Mechanical Engineering, Jiangsu University of Technology, Changzhou \\ 213000, China \\ ${ }^{2}$ Department of Materials, Jiangsu University of Technology, Changzhou 213000, China \\ *Corresponding author's e-mail: yx_laser@163.com
}

\begin{abstract}
After implanting pyrocarbon into human body, patients will have symptoms such as inflammation and thromboembolism, so they must take anticoagulant drugs for life. To improve the blood compatibility of the pyrolytic carbon, a nanosecond laser was used to prepare microstructures on the surface of the pyrolytic carbon. The effects of laser power, scanning times and scanning spacing on the wettability of pyrolytic carbon are discussed. At the same time, dynamic coagulation, hemolysis rate and platelet adhesion test were performed on smooth samples and samples prepared at different scanning spacing. The results show that the contact angle of the modified parallel grating pyrocarbon surface is larger than that of the smooth sample. As the scanning spacing increases, the contact angle increases and then decreases, and the higher the contact angle, the better the blood compatibility of the sample. When the laser power is $27.5 \mathrm{~W}$, the scanning times is 6 , and the scanning distance is $90 \mu \mathrm{m}$, the contact angle of the sample reaches the best, which is $153.21^{\circ}$; the number of platelets adhering to the surface was the least, about 2, and mostly in an unactivated state; the anticoagulant effect is also the best, and the surface hemolysis is the lowest, which is only $0.88 \%$.

DOI: $10.2961 /$ jlmn.2021.01.2010
\end{abstract}

Keywords: nanosecond laser, mechanical heart valve, pyrolytic carbon, superhydrophobicity, blood compatibility

\section{Introduction}

With the development of economy and the extension of life expectancy, China has entered an irreversible aging society. The number of elderly patients with valvular heart disease is gradually increasing, and rheumatic heart disease has gradually evolved into senile degenerative valvular heart disease [1]. At present, 300,000 patients need valve replacement every year, and this number is expected to increase to at least 850,000 by 2050 [2,3]. As far as clinical practice is concerned, the most commonly used replacement valves are biological and mechanical valves [4]. Among them, biological valve has good blood compatibility. However, the dilemma of immunogenicity of animal tissues has not never been solved, and the leaflets of valvular were prone to calcification, which may cause patients to perform secondary valve replacement [5]. Therefore, mechanical heart valve has been occupying the leading position in the market with its excellent durability [6,7].

Pyrolytic carbon was first used clinically as a heart valve material in 1968. The performance test and clinical application of low-temperature pyrocarbon artificial heart valve show that it has good biocompatibility, good anticoagulant, very wear-resistant, invariable, light weight, and meets the clinical requirements [8]. Up to now, pyrocarbon is still the preferred material for mechanical valve. However, compared with the natural heart valve, its biocompatibility is still not ideal. Anticoagulant drugs need to be taken for life after implantation, which brings huge economic burden to patients [9]. Even so, patients will still have symptoms such as inflammation and thromboembolism, so it is of great significance to improve the blood compatibility of artificial mechanical valve and reduce the probability of thrombosis $[10,11]$.

Ikada et al. [12] pointed out that extreme hydrophobic surface has better blood compatibility. With the in-depth research in the field of superhydrophobic, it has been found that superhydrophobic surfaces not only has great advantages in industrial applications and daily life, but also has great potential in biomedicine field. In recent years, some domestic and foreign scholars have built superhydrophobic surfaces on medical materials to improve blood compatibility [13]. Jiang et al. [14] constructed a superhydrophobic coating on the surface of medical titanium alloy by micro-arc oxidation and fluorosilane modification. David et al. [15] used a commercial layered coating on the surface of bilobal mechanical valve and to significantly reduce platelet adhesion. Xiang et al. [16] prepared superhydrophobic coating with simple and rapid casting and soot process, and the cell activity of coating surface was enhanced. Feng et al. [17] prepared bimetallic oxide superhydrophobic surface on a pure magnesium substrate by one-step hydrothermal method. The test result of hemolysis rate showed that the hemolytic rate of pure magnesium was $13.9 \%$, while the hemolysis rate of the superhydrophobic surface is $1.2 \%$, less than $5 \%$, which met the requirements of medical biomaterials. The above results show that superhydrophobic surfaces can improve blood compatibility, but the research on preparation of superhydrophobic surface based on pyrolytic carbon and other non-metallic solid medical materials is less and limited to coating and film [18]. With the passage of time, the adhesion between superhydrophobic coating and substrate is 
insufficient, which will lead to problems such as peeling off or serious wear, and the substrate surface incompatible with blood will be exposed [19].

Superhydrophobic surface refers to the surface with static contact angle greater than $150^{\circ}$, and is mainly prepared by the following two methods: selecting low surface energy materials to construct rough structure or modifying low surface energy materials on rough surface [20]. At present, the conventional methods for preparing superhydrophobic surfaces include laser etching [21-23], chemical etching [24-26], hydrothermal [27,28], spray coating $[29,30]$, and template methods $[31,32]$ et al. Due to the lack of adhesion between the coating, film and substrate, and the difficulty of chemical and oxidation reaction of pyrolytic carbon, laser etching has high stability and good flexibility, which can achieve efficient and controllable construction of regular rough structure on the material surface [33].

In this study, we used a nanosecond laser to etch parallel grating structure on the surface of pyrolytic carbon and modify it with hexadecyltrimethoxysilane (HDTMS). The superhydrophobic pyrolytic carbon surface was prepared by optimizing laser parameters including laser power, scanning spacing and scanning times, which improved blood compatibility. The effects of wettability and microstructure on the blood compatibility of pyrolytic carbon surface was studied by dynamic coagulation, platelet adhesion and hemolysis rate experiments.

\section{Material and methods \\ 2.1 Materials}

Pyrolytic carbon flakes with a dimension of $10 \mathrm{~mm} \times 10$ $\mathrm{mm} \times 2 \mathrm{~mm}$ were used, purchased from Beijing Tengyun Huachang New Material Technology Co., Ltd. The pyrolytic carbon was polished with 600-7000 mesh sandpaper to make the pyrolytic carbon surface smooth, and then cleaned with deionized water and anhydrous ethanol for $30 \mathrm{~min}$, and finally put into the blast drying oven for drying. Hexadecyltrimethoxysilane $\left(\mathrm{H}_{3} \mathrm{C}\left(\mathrm{CH}_{2}\right)_{15} \mathrm{Si}\left(\mathrm{OCH}_{3}\right)_{3}\right)$ was purchased from Shanghai Aladdin Bio-Chem Technology Co., Ltd. Ethanol was purchased from Shanghai Haohai Biotechnology Co., Ltd. Glutaraldehyde $\left(\mathrm{C}_{5} \mathrm{H}_{8} \mathrm{O}_{2}\right)$ was purchased from Fuzhou Brunei Biotechnology Co., Ltd. All chemicals were analytical grade reagents.

\subsection{Fabrication of Pyrolytic carbon Superhydrophobic Surfaces}

As shown in Figure 1(a), the pyrocarbon surface is etched with an infrared laser marking machine (DL-TGIRF-30) along a parallel grating path, and the white solid line is the laser etching position. The repetition frequency of the output laser is $20 \mathrm{kHz}$. The central wavelength is set as $1064 \mathrm{~nm}$, the pulse duration is $100 \mathrm{~ns}$ and the maximum output power is $30 \mathrm{~W}$. The focal length of the lens used is 29 $\mathrm{cm}$. The laser scanning speed is fixed at $100 \mathrm{~mm} / \mathrm{s}$. Superhydrophobic surfaces was prepared by changing laser processing parameters such as laser power, scanning spacing and scanning times, and ultrasonically cleaned with deionized water and ethanol for $30 \mathrm{~min}$, respectively, and dried in a blast drying oven.

The modification scheme of HDTMS on the surface of pyrolytic carbon is shown in Figure 1(b). The laser textured pyrolytic carbon samples were immersed into a hexadecyltrimethoxysilane solution at room temperatures for $60 \mathrm{~min}$ (the hexadecyltrimethoxysilane solution was prepared by adding deionized water, $\mathrm{H}_{3} \mathrm{C}\left(\mathrm{CH}_{2}\right)_{15} \mathrm{Si}\left(\mathrm{OCH}_{3}\right)_{3}$ and ethanol at a mass ratio of $13: 1: 1$, and stirred with a magnetic stirrer for $120 \mathrm{~min}$ ) and dried in a blast drying oven $\left(120^{\circ} \mathrm{C}\right)$ for 120 min to reduce the pyrolytic carbon surface energy.

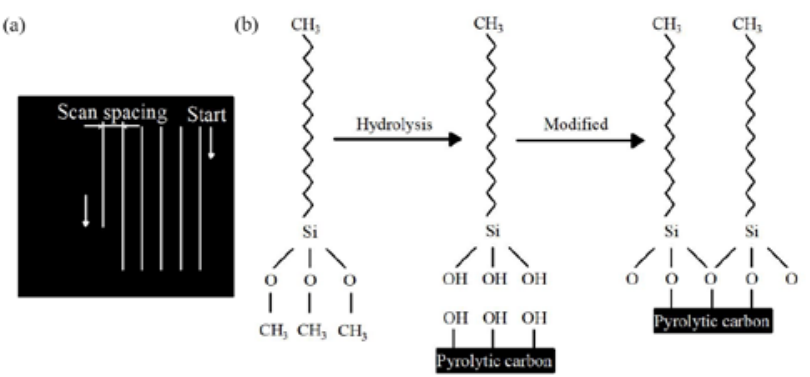

Fig. 1 Sample preparation process.

(a) The trajectory of the laser beam. (b) Modification scheme of HDTMS on pyrolytic carbon surface.

\subsection{Characterization}

Field emission scanning electron microscope (SEM, EVOMA10) is used to observe the pyrolytic carbon surface morphology. The chemical composition and phase composition of the samples were characterized by energy dispersion spectroscopy (EDS) and Fourier Transform Infrared (FTIR). Contact angle was measured by a contact angle measuring instrument (CA100C, Shanghai Innuo Precision Instrument Co., Ltd).

\subsection{Platelet adhesion test}

Platelet-rich plasma (PRP) was obtained by fresh anticoagulated rabbit blood at $1500 \mathrm{rpm}$ for $15 \mathrm{~min}$, the same blood was used in the one experiment. Each sample was placed in PRP and incubated it at $37{ }^{\circ} \mathrm{C}$ water bath for $30 \mathrm{~min}$. Then, the samples were washed with PBS for 3 times. Platelets adhered to the sample surface with immobilized with $2.5 \%$ glutaraldehyde solution at room temperature and washed with phosphate buffer saline (PBS) 2 hours later. Finally, the samples were dehydrated with 30\%, 50\%, 70\%, $90 \%, 100 \%$ ethanol solutions for $20 \mathrm{~min}$, and then dried in air. After spraying gold in vacuum, the samples were observed and photographed by scanning electron microscope (SEM, EVOMA10). In each round of platelet adhesion experiment, five rectangle areas with the same size were randomly selected on the sample surface, and the number of platelets adhered to the surface was counted by ImageJ [34].

\subsection{Dynamic coagulation test}

The sample was first placed in a beaker with $37^{\circ} \mathrm{C}$ water bath for 5 minutes, and then $250 \mu \mathrm{L}$ fresh rabbit blood was dropped onto the surface of the sample with a quantitative pipette. The same batch of blood was used in one experiment. After 5 minutes, $20 \mu \mathrm{L}$ of $0.2 \mathrm{M} \mathrm{CaCl}_{2}$ aqueous solution was injected into the blood and stirred gently. The red blood cells not coagulated on the surface of the sample will have hemolytic reaction, and the free hemoglobin will be dispersed in the water. Finally, the absorbance of the solution was measured by spectrophotometer at $540 \mathrm{~nm}$. 


\subsection{Hemolysis test}

Diluted anticoagulated rabbit blood was obtained by mixing $4 \mathrm{ml}$ anticoagulated blood with $5 \mathrm{ml}$ normal saline, the same batch of blood was used in one experiment. The sample to be tested was placed in a glass container, and 10 $\mathrm{ml}$ of physiological saline was added. After incubation in 37 ${ }^{\circ} \mathrm{C}$ water bath for $30 \mathrm{~min}, 200 \mu \mathrm{L}$ diluted anticoagulant rabbit blood was added. After $60 \mathrm{~min}$, the solution was centrifuged at $1000 \mathrm{rpm}$ for $5 \mathrm{~min}$. The absorbance of the supernatant fluid of the solution was measured by spectrophotometer at $540 \mathrm{~nm}$. The positive reference was $200 \mu \mathrm{L}$ blood and $10 \mathrm{ml}$ water, and the negative reference was $200 \mu \mathrm{L}$ blood and $10 \mathrm{ml}$ normal saline. Finally, the percentage of hemolysis was evaluated according to the following equation (Eq. (1)) [35]:

Hemolysis $(\%)=\frac{D_{\mathrm{t}}-D_{n c}}{D_{p c}-D_{n c}} \times 100 \%$

where $D_{t}$ is the absorbance value of the samples, $D_{p c}$ and $D_{n c}$ was the absorbance value of positive control and negative control.

\section{Results and discussion \\ 3.1 Surface morphology}

Figure 2 shows the surface morphology of parallel grating structure prepared by pyrolytic carbon at different scanning spacing. Figure 2 (a) shows the surface morphology of polished smooth pyrocarbon. It can be seen from the figure that the surface of the polished sample is relatively smooth and flat, with some scratches randomly distributed. From the enlarged images of some regions, it can be seen that the smooth pyrolytic carbon surface presents a layered microscopic morphology. The laser power is $27.5 \mathrm{~W}$ and the scanning times are 6 times. The parallel grating structure is fabricated by laser beam etching on the smooth pyrolytic carbon surface line by line. When the laser beam irradiates the pyrolytic carbon surface, the high heat will propagate along the material surface due to the photothermal effect, which will cause the surface to melt or vaporize. With the laser beam moving, the processing area will cool rapidly and form regular structure on the surface. When the laser power is low, the energy of the etching region is low and etching rate is slow. Therefore, the variation of the depth and width of the groove is relatively small. With the increase of scanning times, the depth of grooves increases to a certain extent, and the heat affected zone of laser beam becomes smaller. Therefore, the depth of the material etched in the center of the laser beam will be significantly greater than the depth of the edge, thus forming a slope.

It can be seen from Figure 2 that under the selected laser parameters, the maximum width of the thermal influence of the laser beam is about $55 \mu \mathrm{m}$. As shown in Figure 2 (b), when the laser scanning spacing is $60 \mu \mathrm{m}$, the reserved grating, that is, the width of the transition region between the grooves, is too small, and the grating is partially broken. Compared with the complete grating, the incomplete fracture part will be slightly tilted. It can also be seen from the figure that the completely fractured part is only a layer of surface with nanoparticles falling off, exposing the original smooth surface. From the enlarged Figure 2(f), it can be found that the whole grating part has a large number of protrusions similar to the hemispherical structure of corn kernels. These nanoparticles fuse with each other, and there are some pores between them, which combine with the grating microstructure to form micro nano composite structure on the surface of pyrolytic carbon.

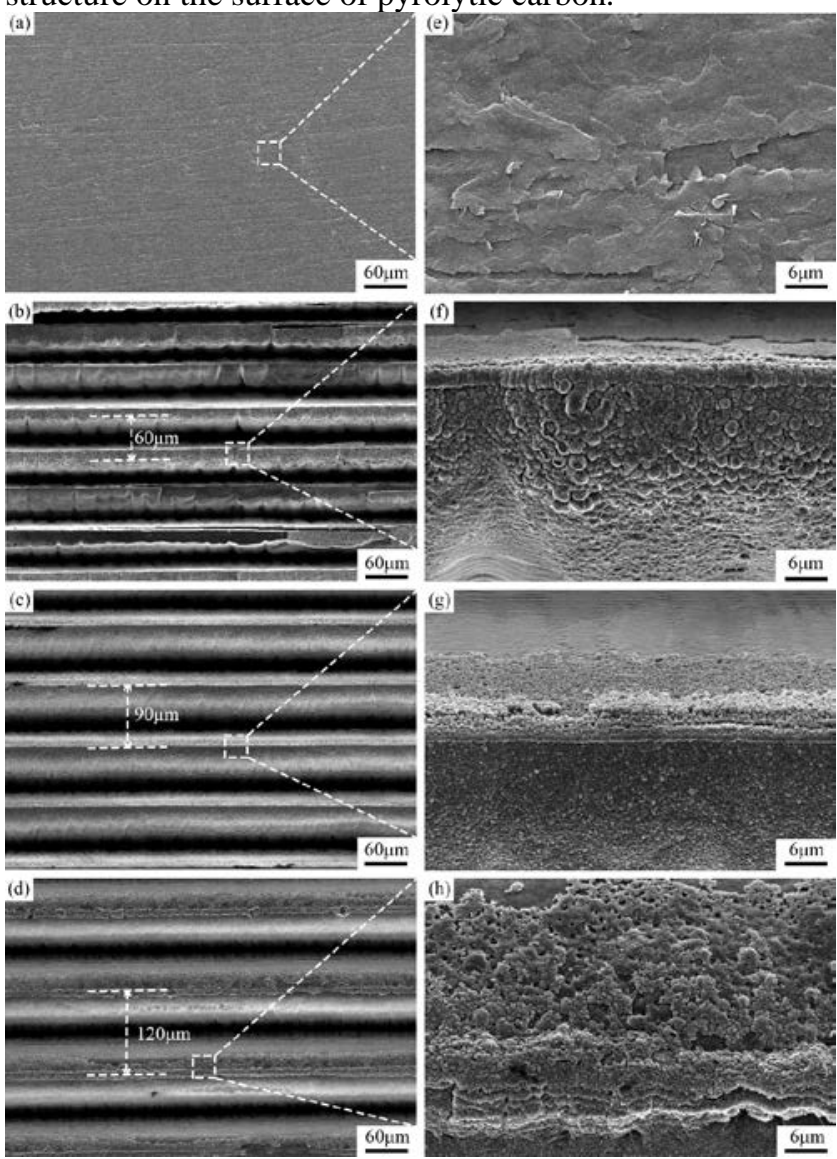

Fig. 2 Surface topography of samples prepared at different scanning spacing.

As the laser scanning spacing increases to $90 \mu \mathrm{m}$, as shown in Figure 2(c), at low magnification, it is found that the number of parallel gratings on pyrocarbon surface decreases and becomes more regular, and the grating fracture phenomenon no longer exists. At high magnification, it is found that there are a large number of nanoparticles in the transition region of the groove, and the multilayer nanoparticles are stacked at the edge of the grating structure. The nanoparticles on the surface are almost spherical particles with small diameter and dense distribution. At the same time, more and smaller pores are formed on the surface. Part of the micron and nanometer particles may be caused by the sputtering of the melted material in the laser etching area to other parts after cooling, and may also be attributed to the local material movement caused by the Marangoni [36]. When the laser scanning spacing increases to $120 \mu \mathrm{m}$, the parallel grating structure is still regular, and the width of the transition region between the grooves increases again. At the edge of the grating structure, the nanoparticles are stacked to form a long spine about $3 \mu \mathrm{m}$ high and $5 \mu \mathrm{m}$ wide. It can also be found from the local enlarged image that these spherical nanoparticles are closely connected with the layered structure, forming macropores where the nanoparticles gather, and some small holes where the nanoparticles are sparse. It is found that the 
microstructure of the sample surface changes from a large number of nano particles to a smooth slope, and then to a stepped wavy structure. Finally, when the groove is too deep, the bottom of the groove cannot be seen. The difference of microstructure will affect the contact angle of pyrolytic carbon surface, which will be discussed later.

\subsection{Surface wettability}

In order to evaluate the change of the wettability of the sample surface, the contact angle of the smooth pyrolytic carbon surface, the modified smooth pyrolytic carbon surface, the microstructured pyrolytic carbon surface after laser etching, the laser-etched and modified pyrolytic carbon surface were measured. As seen in Figure 3(a), the intrinsic contact angle of the smooth pyrolytic carbon surface is about $88^{\circ}\left(<90^{\circ}\right)$, indicating that the untreated pyrolytic carbon surface is hydrophilic. After HDTMS modification, the contact angle increases to $103^{\circ}$, indicating that the modified pyrolytic carbon is enhanced in hydrophobicity due to the reduction of surface energy. Only nanosecond laser was used to prepare microstructures on the pyrolytic carbon surface, the surface is superhydrophilic, and the

(a)

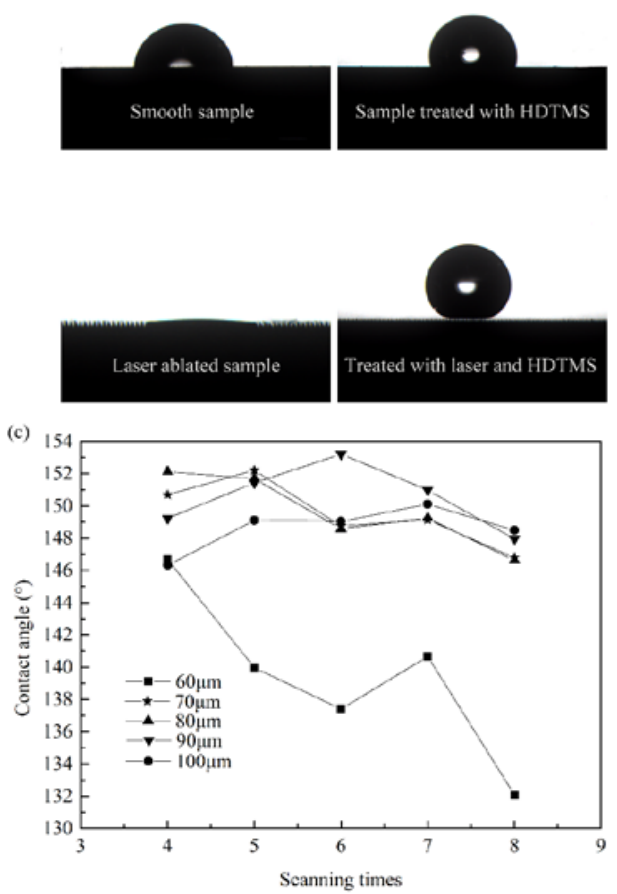

contact angle is small, which is difficult to measure. The results show that the samples with optimized laser parameters and silanization can reach superhydrophobic state.

Figure 3(b) shows the effect of laser power and scanning times on the wettability of pyrolytic carbon. As shown in Figure 3(b), the fixed scanning spacing is $70 \mu \mathrm{m}$, the scanning times and laser power range are 4-8 times and 15$30 \mathrm{~W}$ respectively. When the laser power is small, (e.g. 15 $\mathrm{W})$, the contact angles of the samples are smaller than $150^{\circ}$ in this range. When the laser power is too high, the contact angle first increases and then decreases rapidly with the increase of scanning times. By comparison, it is found that the number of scans needed to reach superhydrophobic state is less at high power. The results show that with the increase of laser power, it is easier for the laser beam to ablate the pyrolytic carbon surface. Under the same scanning times, the thermal effect area of laser spot is larger and the groove depth is deeper. However, with the increase of scanning times, the depth of laser etching is limited, and subsequent etching may damage the prepared microstructure, resulting in the decrease of contact angle.
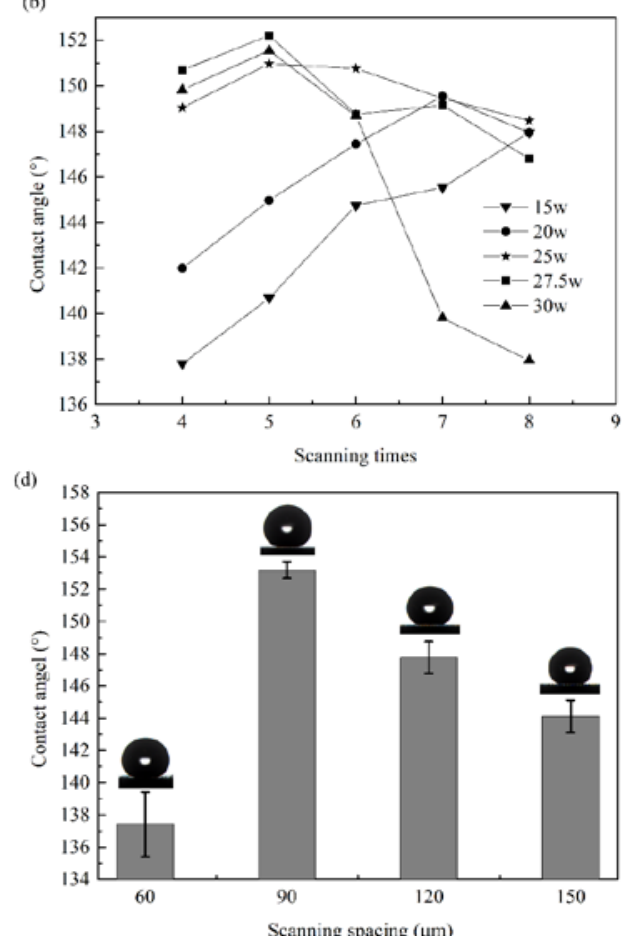

Fig. 3 Change of water contact angle on pyrolytic carbon surface. a) water contact angles of pyrolytic carbon surface; b) effect of laser power and scanning times on surface wettability; c) effect of scanning times and scanning spacing on surface wettability; d) effect of scanning spacing on surface wettability when the laser power and the scanning times were set as $27.5 \mathrm{~W}$ and 6 times.

Figure 3(c) shows the effect of scanning spacing and scanning times on the wettability of pyrolytic carbon. By analyzing the change of the contact angle in Figure 3(b), the laser power is selected to be $27.5 \mathrm{~W}$, and the scanning spacing is changed between 4-8 scanning times, as shown in Figure 3(c). It is found that when the scanning spacing is small, such as $60 \mu \mathrm{m}$, the contact angle of the sample is small and decreases with the increase of scanning times. When the laser power is $27.5 \mathrm{~W}$, the diameter of the spot is about $55 \mu \mathrm{m}$. When the scanning spacing is small, the grating width is too small, which is easy to fracture in the process of processing, resulting in damage to the groove structure. After increasing the scanning spacing, the grating fracture phenomenon no longer exists, and the samples begin to show superhydrophobic. When the laser power is $27.5 \mathrm{~W}$, the scanning times is 6 , the scanning spacing is 90 $\mu \mathrm{m}$, and the contact angle is $153.21 \pm 0.5^{\circ}$. It can be concluded that the combined action of micro nano structure and low surface energy leads to the formation of superhydrophobicity of pyrolytic carbon in the air, which 
can be explained by Cassie-Baxter model [37-38]. In the air, the cavitation is trapped in the gap of rough surface, and the water droplet only contacts with the top of the groove, forming a very discontinuous three-phase contact interface, which lead to the increase of contact angle. When the scanning spacing continues to increase, the contact angle shows a downward trend. This is because when the laser power is constant and the scanning distance is increased, the diameter of the spot is a constant value. From the cross section, the width of the groove does not change, but the width of the smooth plane that is not etched increases. When the liquid contacts the sample, the solidliquid contact area increases, and the contact state of the droplet on the sample surface changes from Cassie-Baxter to Wenzel. At this time, increasing the depth of the groove will increase the air between the droplet and the groove, so that the liquid can maintain solid-liquid-gas three-phase contact on the sample surface. Therefore, after increasing the scanning spacing, it is necessary to increase the scanning times to increase the depth of the groove, so as to keep the Cassie-Baxter state on the sample surface.

The selected laser power and scanning times are $27.5 \mathrm{~W}$ and 6 times respectively to fix the depth and width of the groove structure. Only the influence of scanning spacing on the contact angle is discussed, as shown in Figure 3(d). With the increase of scanning spacing, the contact angle first increases and then decreases. This is because when the depth and width of the groove are fixed, the droplets are in a stable Cassie state on the surface of the sample within a certain range, and cannot enter into the groove. With the decrease of the ratio of the groove width to the scanning spacing, the microstructure prepared by laser etching will be more regular and will not be warped or broken due to the small scanning distance. Regular nanostructures will reduce the solid-liquid contact area and increase the contact angle. However, when the ratio continues to increase, the droplet will be in the transition region from Cassie to Wenzel. The droplet will penetrate into the groove, thus increasing the solid-liquid contact area, and the contact angle will no longer increase but tend to decrease. When it is completely in Wenzel state, the contact angle decreases with the decrease of the ratio. The above results show that when the scanning spacing is fixed, the contact angle of the sample can be increased by increasing the laser power, reducing the scanning times and selecting the appropriate scanning times, or reducing the width of the reserved grating without damaging the overall morphology of the sample microstructure.

\subsection{Chemical composition}

According to the modification scheme shown in Figure 1(b), after the hydrolysis of HDTMS, the methoxy group is replaced by hydroxyl group. The sample and the hydroxyl groups in the solution undergo a dehydration condensation reaction to form a covalent bond, and finally a hydrophobic film is formed on the sample surface. Table 1 shows the composition changes of EDS on the sample surface. In Table 1 , the main components of pyrolytic carbon are $\mathrm{C}$ and $\mathrm{O}$.

After laser etching, no new elements appear on the surface of the sample. Compared with smooth pyrolytic carbon, only the carbon content decreases and the oxygen content increases. After modification with HDTMS, a small amount of Si appeared on the sample surface, and the mass fraction of Si was only $0.2 \%$. This indicates that neither laser etching nor HDTMS modification can react with pyrolytic carbon, and HDTMS has been successfully modified on the surface of pyrolytic carbon.

Table 1 EDS of pyrolytic carbon surfaces at different treatment stages

\begin{tabular}{cccc}
\hline Treatment & \multicolumn{3}{c}{ Quality score /\% } \\
\cline { 2 - 4 } stage & $\mathrm{C}$ & $\mathrm{O}$ & $\mathrm{Si}$ \\
\hline $\begin{array}{c}\text { Smooth } \\
\text { sample }\end{array}$ & 95.6 & 4.4 & $/$ \\
Laser ablated & 88.5 & 11.5 & $/$ \\
sample & & & \\
Treated with & & & \\
laser and & 88 & 11.8 & 0.2 \\
HDTMS & & & \\
\hline
\end{tabular}

After laser etching, no new elements appear on the surface of the sample. Compared with smooth pyrolytic carbon, only the carbon content decreases and the oxygen content increases. After modification with HDTMS, a small amount of Si appeared on the sample surface, and the mass fraction of Si was only $0.2 \%$. This indicates that neither laser etching nor HDTMS modification can react with pyrolytic carbon, and HDTMS has been successfully modified on the surface of pyrolytic carbon.

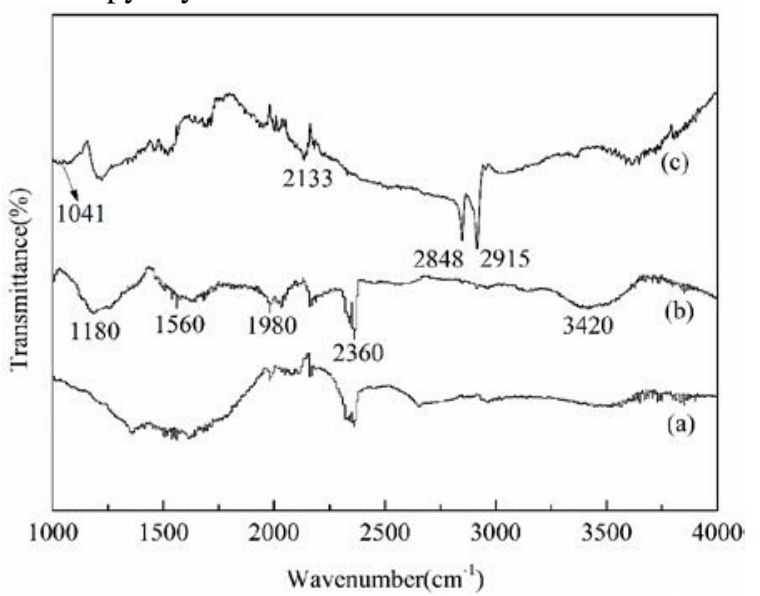

Fig. 4 FTIR spectra under the surface of pyrolytic carbon in different treatment stages.

In Figure 4, curve a, curve b and curve c are the infrared absorption spectra of smooth pyrolytic carbon surface, laser etched pyrolytic carbon surface, and laser etched pyrolytic carbon surface modified with HDTMS, respectively. From curves a and b in Figure 4, it can be found that the chemical composition and structure of the pyrolytic carbon surface after laser etching have not changed. Among them, there is a strong C-O-C absorption peak at $1180 \mathrm{~cm}^{-1}$. There are $\mathrm{C}=\mathrm{C}$, $\mathrm{C}=\mathrm{O}$ and $\mathrm{C} \equiv \mathrm{C}$ absorption peaks at $1560 \mathrm{~cm}^{-1}, 1980 \mathrm{~cm}^{-1}$ and $2360 \mathrm{~cm}^{-1}$, respectively. The peak at $3420 \mathrm{~cm}^{-1}$ is the $\mathrm{O}-\mathrm{H}$ absorption peak. However, after the laser-etched pyrolytic 
carbon modified by HDTMS, the infrared absorption spectrum of the surface has undergone major changes. As is shown in the curve of Figure 2c, there is no $\mathrm{C} \equiv \mathrm{C}$ absorption peak at $2360 \mathrm{~cm}^{-1}$, while the $\mathrm{Si}-\mathrm{O}-\mathrm{Si}$ absorption peak appears at $1041 \mathrm{~cm}^{-1}$, and the $\mathrm{Si}-\mathrm{H}$ absorption peak appears at $2133 \mathrm{~cm}^{-1}$, indicating that the HDTMS molecules have reacted during the process of binding to the surface. C-H absorption peaks were found in both $2848 \mathrm{~cm}^{-1}$ and 2915 $\mathrm{cm}^{-1}$. The results of infrared spectroscopy show that HDTMS is successfully modified on the surface of pyrolytic carbon, and the increase of the hydrophobic group $\mathrm{CH}_{3}$ makes the surface of pyrolytic carbon superhydrophobic.

\subsection{Hemocompatibility}

Pyrolytic carbon is commonly used as a material for
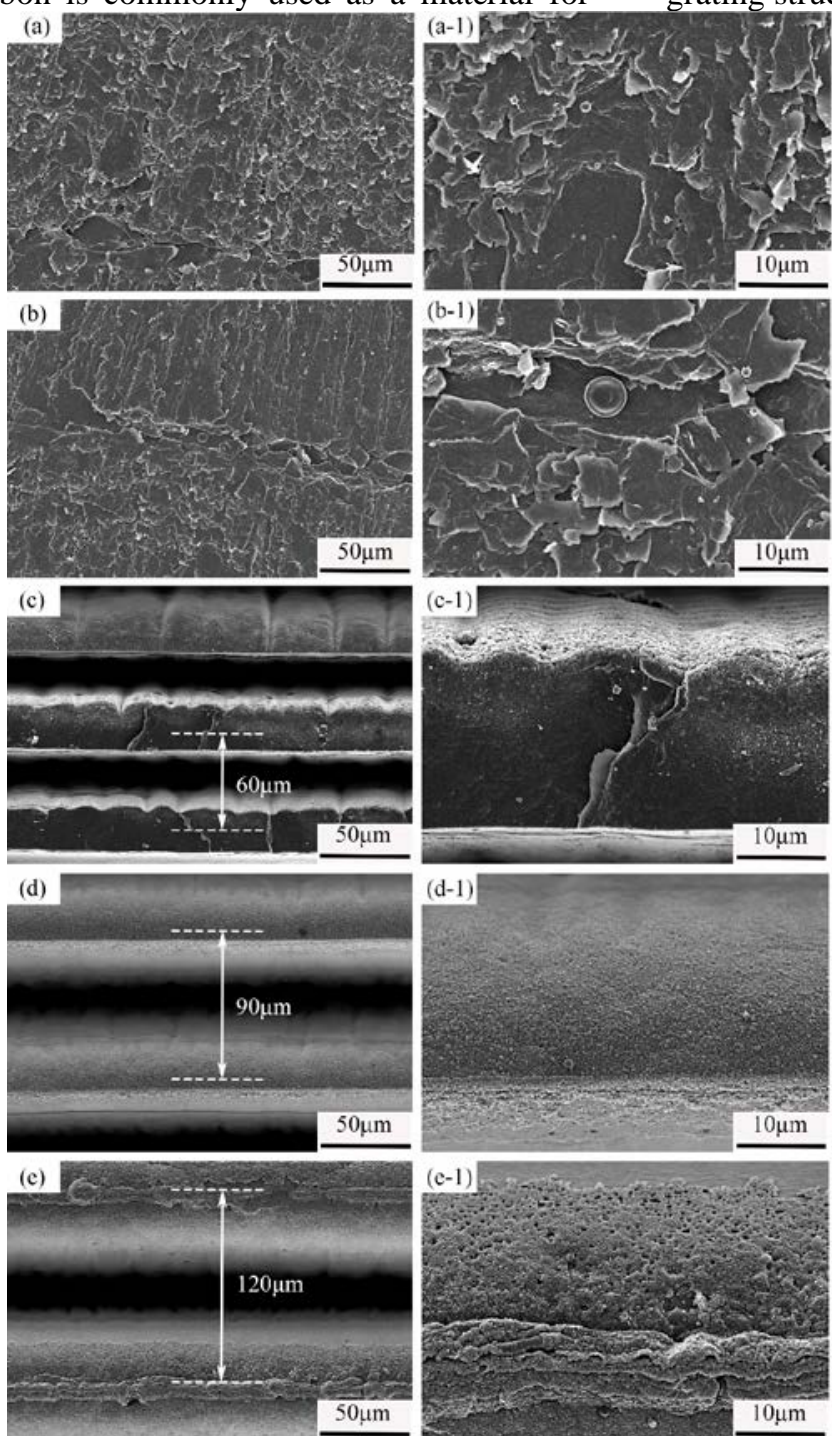

Fig. 5 SEM image of platelet adhesion on the various pyrolytic carbon samples

(a) Pyc (b) Hpyc (c) PGS-60 $\mu \mathrm{m}$ (d) PGS-90 $\mu \mathrm{m}$ (e) PGS-120 $\mu \mathrm{m}$.

Figure 5 is the SEM image of platelet adhesion on the various pyrolytic carbon samples. The contact angles of Pyc, Hpyc, PGS-60 $\mu \mathrm{m}, \mathrm{PGS}-90 \mu \mathrm{m}$ and PGS-120 $\mu \mathrm{m}$ are $88^{\circ}$, $103.42^{\circ}, 137.41^{\circ}, 153.21^{\circ}, 147.76^{\circ}$; (a-1), (b-1), (c-1), (d-1), (e-1) are surface topography of Pyc, Hpyc, PGS-60 $\mu \mathrm{m}$, PGS-90 $\mu \mathrm{m}$, PGS-120 $\mu \mathrm{m}$ of platelet adhesion. It can be seen from the scanning electron microscope that the number of platelets adhering to the smooth pyrolytic carbon surface is the largest. After HDTMS modification, a large number of platelets still adhere to the smooth pyrolytic carbon surface, which shows that the HDTMS modification does not reduce the sample surface anticoagulant performance. From the enlarged view of the platelets on the Pyc surface in Figure 5 (a-2), it can be found that the shape of part platelet in the smooth pyrolytic carbon surface changes irreversibly. From the enlarged view of red blood cells on 
JLMN-Journal of Laser Micro/Nanoengineering Vol. 16, No. 1, 2021

the surface of Hpyc in Figure 5 (b-2), it can be seen that the red blood cells attached to the surface of the pyrolytic carbon are not activated and they are disc-shaped. However, the number of platelets adhering to the surface of the parallel grating pyrolytic carbon significantly decreased and most of them were not activated. This is because the micro-nano composite structure on the surface of the pyrolytic carbon not only effectively reduces the effective area of the sample exposed to platelets, but also reduces the available adhesion area of individual platelets. It can be seen from Figure 5(c) that due to the small width of the grating reserved for PGS$60 \mu \mathrm{m}$, some grating areas are fractured, and platelets mainly adhere to the smooth surface and fracture after fracture. It can be seen from Figure 5(c-2) that although some platelets do not extend pseudopodia, there is platelet aggregation at the fracture site. With the increase of scanning spacing, the microstructure becomes regular, and the PGS-90 $\mu \mathrm{m}$ surface is covered with a large number of spherical particles. These nanoparticles and the pores between them mostly smaller than the diameter of platelets, which makes the actual contact area between platelets and sample surface smaller. With the increase of contact angle, it can also be found from Figure 5 (d-1) and Figure 5 (d-2) that the number of platelets adhering to the surface of PGS$90 \mu \mathrm{m}$ is the least, and the platelets are disc-shaped. This indicates that the platelets are not activated and can still play the role of coagulation, hemostasis and repair of damaged blood vessels. When the scanning spacing continues to increase, the spherical granular carbon structure on the sample surface is stacked repeatedly, and many large-scale pores are formed on the sample surface. From Figure 5 (e-1) and Figure 5 (e-2), it can be seen that these pores are larger than the diameter of platelets. When the platelets adhere to the pores, they will produce pseudopodia. When the platelets adhere to other parts, most of them remain inactive.

Table 2 shows the number of platelets adhering to the surface of Pyc, Hрyc PGS-60 $\mu \mathrm{m}$, PGS-90 $\mu \mathrm{m}$, and PGS$120 \mu \mathrm{m}$ samples. The statistical area is about $2536 \mu \mathrm{m}^{2}$. After soaking in the PRP solution for $30 \mathrm{~min}$, the number of platelets adhering to the smooth pyrolytic carbon surface was about 11 at most. The number of platelets adhering to the pyrolytic carbon of parallel grating decreased. The number of platelets adhering to the surface of Hpyc, PGS$60 \mu \mathrm{m}$, PGS-90 $\mu \mathrm{m}$ and PGS-120 $\mu \mathrm{m}$ samples was about 10 , 7, 2 and 4, respectively. Combined with the SEM image of platelet adhesion on the various pyrolytic carbon samples, compared with platelets on a smooth pyrolytic carbon surface, the number of platelets adhering to the parallel grating pyrolysis carbon surface all showed a downward trend. This is because the sample surface is covered by a large number of micro and nano particles. The gap between these nanoparticles is small enough that platelets can not adhere and can only exist on the top of the nanoparticles. Thus, the usable adhesion area of a single platelet is reduced. With the increase of scanning spacing, the number of platelet adhesion on the parallel grating pyrocarbon surface decreases with the increase of contact angle. This is because the stronger the hydrophobicity of the sample surface, the lower the surface energy, and the stronger the anti-platelet adhesion ability. when the sample reaches superhydrophobic property, the blood and sample will be in Cassie state, which will reduce the actual contact area between blood and sample and improve the anticoagulant property of Рyc surface.

Table 2 Number of adherent platelets on the various samples

\begin{tabular}{ccc} 
Sample & Contact angle $\left(^{\circ}\right)$ & Number of platelet \\
Pyc & $88^{\circ}$ & 11 \\
Hpyc & $103.42^{\circ}$ & 10 \\
PGS-X & $130 \mu \mathrm{m}$ & 7 \\
PGS-X $_{90 \mu \mathrm{m}}$ & $153.21^{\circ}$ & 2 \\
PGS-X $_{120 \mu \mathrm{m}}$ & $147.76^{\circ}$ & 4 \\
\hline
\end{tabular}

When Pyc comes into contact with blood, soluble fibrinogen will become insoluble fibrin filament under different mechanisms. These filaments interweave and aggregate, which will adsorb platelets, white blood cells, red blood cells and other tangible components to form thrombus and cause coagulation. Therefore, platelets are active on the surface of the material, and the ability of the material to resist endogenous coagulation is judged by the absorbance value of uncoagulated blood after contacting with the sample for a certain period of time.

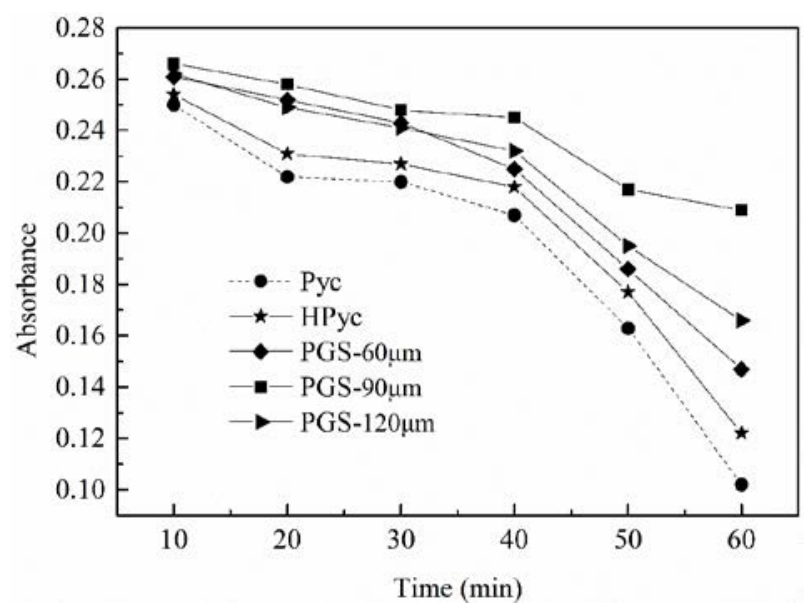

Fig. 6 Kinetic clotting time curves on the various samples

Figure 6 shows the dynamic clotting time curve of each sample. It can be found that with the increase of contact time between the sample and blood, the absorbance shows a continuous downward trend. This is because with the increase of time, the degree of blood coagulation on the surface of the sample increases, and the number of red blood cells in the blood decreases. Among them, the absorbance value of anticoagulant blood in contact with the surface of Pyc decreased rapidly, while the absorbance value of anticoagulant blood in contact with PGS-90 $\mu \mathrm{m}$ decreased the slowest, and the corresponding absorbance value of each time period was the largest. This means that the number of red blood cells in the blood contacting with PGS-90 $\mu \mathrm{m}$ is the largest, and PGS-90 $\mu \mathrm{m}$ has the best anticoagulant effect. It can also be seen from Figure 6 that with the increase of coagulation time, the difference of anticoagulation between different samples becomes more and more obvious. The 
absorbance values of Pyc, HPyc, PGS-60 $\mu \mathrm{m}$, PGS-90 $\mu \mathrm{m}$ and PGS-120 $\mu \mathrm{m}$ samples were 0.102, 0.122, 0.147, 0.209 and 0.166 respectively when the dynamic coagulation time was $60 \mathrm{~min}$. This indicates that it is limited to improve anticoagulant properties only by reducing the surface energy of pyrocarbon. The surface of superhydrophobic pyrolytic carbon with microstructure can significantly improve the anticoagulant property, and the anticoagulant property is closely related to the wettability. Generally, the higher the contact angle of the sample, the better the anticoagulant performance. This is mainly because the construction of microstructure and the reduction of surface energy not only enhance the hydrophobicity and reduce the adhesion, but also greatly reduce the contact area between the blood and the sample surface due to the air layer between the microstructure. Thus, the dynamic coagulation time is prolonged and the anticoagulant property is improved.

Table 3 The hemolysis rate of pyrolytic carbon samples

\begin{tabular}{cccc}
\hline Sample & $\begin{array}{c}\text { Contact } \\
\text { angle }\left(^{\circ}\right)\end{array}$ & $\begin{array}{c}\text { Value of } \\
\text { absorbance }\end{array}$ & $\begin{array}{c}\text { Hemolysis } \\
\text { rate (\%) }\end{array}$ \\
\hline Pyc & 88 & 0.0581 & $4.89 \%$ \\
HPyc & 103.42 & 0.0564 & $4.4 \%$ \\
PGS-60 $\mu \mathrm{m}$ & 137.41 & 0.0469 & $1.61 \%$ \\
PGS-90 $\mu \mathrm{m}$ & 153.21 & 0.0444 & $0.88 \%$ \\
PGS-120 $\mu \mathrm{m}$ & 147.76 & 0.0454 & $1.17 \%$ \\
Positive & $/$ & 0.3823 & $/$ \\
control (D) & & & $/$ \\
Negative & $/$ & 0.0414 & \\
control (Dnc & & &
\end{tabular}

When the material comes into contact with the blood, the red blood cells in the blood break and release hemoglobin, which is called hemolysis. After blood and material contact for a period of time, hemoglobin concentration can be reflected by absorbance value, and then converted into hemolysis rate. In this process, broken red blood cells will cause platelets to deform and cause coagulation. Hemolysis rate can directly and reliably reflect the effect of material on red blood cells, so hemolysis rate test is also an important index to evaluate the blood compatibility of materials. The hemolysis rate values of various samples are compared in Table 3. The hemolysis rate of pyrolytic carbon is less than $5 \%$, which meets the requirements of medical biomaterials. By using nanosecond laser to prepare parallel grating superhydrophobic structure on the smooth pyrolytic carbon surface, the hemolysis rate of the sample can be reduced. With the increase of scanning spacing, hemolysis rate first decreased and then increased. When the scanning spacing was $90 \mu \mathrm{m}$, the hemolysis rate was the lowest, only $0.88 \%$, which was consistent with the results of platelet adhesion experiments and dynamic coagulation experiments.

\section{Conclusion}

The result show that by the superhydrophobic surface can be prepared on pyrolytic carbon by nanosecond laser etching and HDTMS modification. We discussed the influence of nanosecond laser parameters on the wettability of pyrolytic carbon in detail, and found that it takes less scanning times to etch pyrolytic carbon into superhydrophobic state at high power. When the scanning spacing was is only increased, the contact angle first increases and then decreases. Through the hemolysis rate, platelet adhesion and dynamic coagulation experiments, we proved that the blood compatibility and anticoagulant properties of the parallel grating microstructure surface increased with the increase of contact angle compared with the untreated smooth surface. PGS-90 $\mu \mathrm{m}$ surface can reduce platelet adhesion and activation and has the lowest hemolysis rate and excellent blood compatibility. This is because the construction of the microstructure and the reduction of surface energy enhance the superhydrophobicity and reduce the adhesion; on the other hand, the cavitation is trapped in the gap of the surface microstructure, and the blood only contacts the top of the groove, forming a very discontinuous three-phase contact interface, greatly reducing the contact area between blood and pyrolytic carbon.

\section{Acknowledgment}

This work was supported by the National Natural Science Foundation of China (No. 11872026), the Natural Science Foundation of Jiangsu Higher Education Institutions of China (No. 18KJA460003) and the National Key Research and Development Program of China (2020YFC2004404).

\section{References}

[1] X.J. Jin, J.S. Wang, and H.Z. Chen: Chinese. J. Chin. Med., 4, (2001) 329. (in Chinese)

[2] H.B. Eva, J.H. Butt, N.E. Vinding, T.P. Christian, G. Gunnar, K. Lars, and L.F. Emil: J. Thoarc. Cardivo. Sur., 159, (2020) 74.

[3] M.H. Yacoub and J.J.M Takkenberg: Nat. Clin. Pract. Card., 2, (2005) 60.

[4] P. Pibarot and J.G. Dumesnil: Circulation, 119, (2009) 1034.

[5] C. Nancy, P. Philippe, and M.A. Clavel: Curr. Opin. Card., 21, (2017) 123.

[6] W. Elsmari, J.J. Christiaan, B. Lezelle, and E.S. Francis: Asian. Card. Thora. Annals., 7, (2019) 1.

[7] A.D.W. Richard, Q. Naureen, and L.Carr: Ann. Thorac. Surg., 65, (2000) 1612.

[8] V.O. Björk: Ann. Thorac. Surg., 50, (1990) 151.

[9] M. Sarah, H.l. Jaffer, P.B. Emilie, R.M. Graham, W.E. John, and P.W. Richard: Circulation, 140, (2019) 1933.

[10] H. Zhang, Y.J. Dong, X.L. Ao, Z. Da, and L. Dong: Eur J. Pharm. Sci., 144, (2020) 105202.

[11] Søndergaard, Lars, Saraste, Antti, Christersson, Christina, Vahanian, and Alec: Eur. Heart. J., 39, (2018) 650.

[12] Y. Ikada: Adv. Polym. Sci., (1984) 103.

[13] T.L. Sun, H. Tan, D. Han, Q. Fu, and L. Jiang: Small, 10, (2005) 959.

[14] J.Y. Jiang, H.Y. Liu, P. Zhang, and T.W. Zhang: Rare. Metal. Mat. Eng., 48, (2019) 1884. (in Chinese)

[15] L.B. David, V. Hamed, B. Hieu, M. Sanli, M. Brandon, 
K.K. Arun, P. Ketul, and P.D. Lakshmi: Ann. Biomed. Eng., 45, (2017) 452.

[16] X.D. Lin, S. Park, D. Choi, J. Heo, and J. Hong: J. Ind. Eng. Chem., 74, (2019)79.

[17] F. Peng, D.H. Wang, X.H. Ma, H.Q. Zhu, Y.Q. Qiao, and Y.X. Liu: Sci. China. Mater., 61, (2018) 629.

[18] E. Salimi: Int. J. Polym. Mater., 69, (2020) 363.

[19] J. Ville, K. Esko, H. Sasha, F. Sami, and H.A.R. Robin: Adv. Mater., 30, (2018) 1705104.

[20] D. Quéré: Rep. Prog. Phys., 68, (2005) 2495.

[21] Y.J. Tuo, H.F. Zhang, W.T. Rong, S.Y. Jiang, W.P. Chen, and X.W. Liu: Langmuir, 35, (2019) 11016.

[22] J. Li, Y.J. Zhou, W.B. Wang, C.Y. Xu, and L.Q. Ren: Langmuir, 36, (2020) 1075.

[23] H. Yang, K.C. Xu, C.W. Xu, D.Y. Fan, C. Yu, X. Wei, and J.H. Pang: Nanoscale. Res. Lett., 14, (2019) 333.

[24] R.A. Mohammad, K. Ehsan, H. Saman, and D. Ali: B. Mater. Sci., 43, (2020) 31.

[25] X. Zhang, J. Zhao, J.L. Mo, R.Y. Sun, L. Zhen, and Z.G. Guo: Colloid. Surface. A., 567, (2019) 205.

[26] A.S. Tawfix and B. Nadeem: Prog. Org. Coat., 133, (2019) 27.

[27] B.B. Zhang, J. Wang, and J. Zhang: Colloid. Surface.
A., 589, (2020) 124469.

[28] S.S. Raad and K.F. Nisreen: J. Adhes. Sci. Technol., 33, (2019) 1558.

[29] H.J. Qian, M.L. Zhu, H. Song, H.Y. Wang, Z.J. Liu, and C.J. Wang: Prog. Org. Coat., 142, (2020) 105566.

[30] T.S. Bhuvaneswari, P.A. Jaya, S.E. Jean, and F.N. Nishad: J. Clean. Prod., 256, (2020) 120693.

[31] W.T. Xu, P.Y. Yi, J. Gao, Y.J. Deng, L.F. Peng, and X.M. Lai: ACS Appl. Mater. Inter., 2, (2020) 12.

[32] R. Simonetta and L. Marco: Small, 14, (2018) 1802854.

[33] X. Wang, B. Xu, Y. Chen, C. Ma, Y. Huang: Opt. Laser. Eng., 122, (2019) 319.

[34] A.H. Md, K. Preoyati, C.C. Lu, and J.Robert: IET. Image. Process., 14, (2020) 2937.

[35] M. Fatemeh, G. Nasim, K. Mahshid, and A. Ali: Int. J. Biol. Macro., 127, (2019) 159.

[36] N. V. Alexey and V.Z Leonid: Int. J. Heat. Mass. Tran., 112, (2017) 300.

[37] R.N. Wenzel: Ind. Eng. Chem. Res., 28, (1936) 988.

[38] A.B.D. Cassie and S. Baxter: Trans. Faraday. Soc., 40, (1944) 546.

[39] J.H. Zhang and X. Chen: Key. Eng. Mat., 464, (2011), 749.

(Received: November 22, 2020, Accepted: February 3, 2021) 